\title{
Produtos alimentícios à base de extrato de Cannabis: do "Brisadeiro" à busca por efeito terapêutico
}

\author{
Food products based on Cannabis extract: from "Brisadeiro" to searching for a therapeutic effect \\ Productos alimenticios a base de extracto de Cannabis: del "Brisadeiro" a la búsqueda del efecto \\ terapéutico
}

Recebido: 06/01/2022 | Revisado: 10/01/2022 | Aceito: 18/01/2022 | Publicado: 19/01/2022

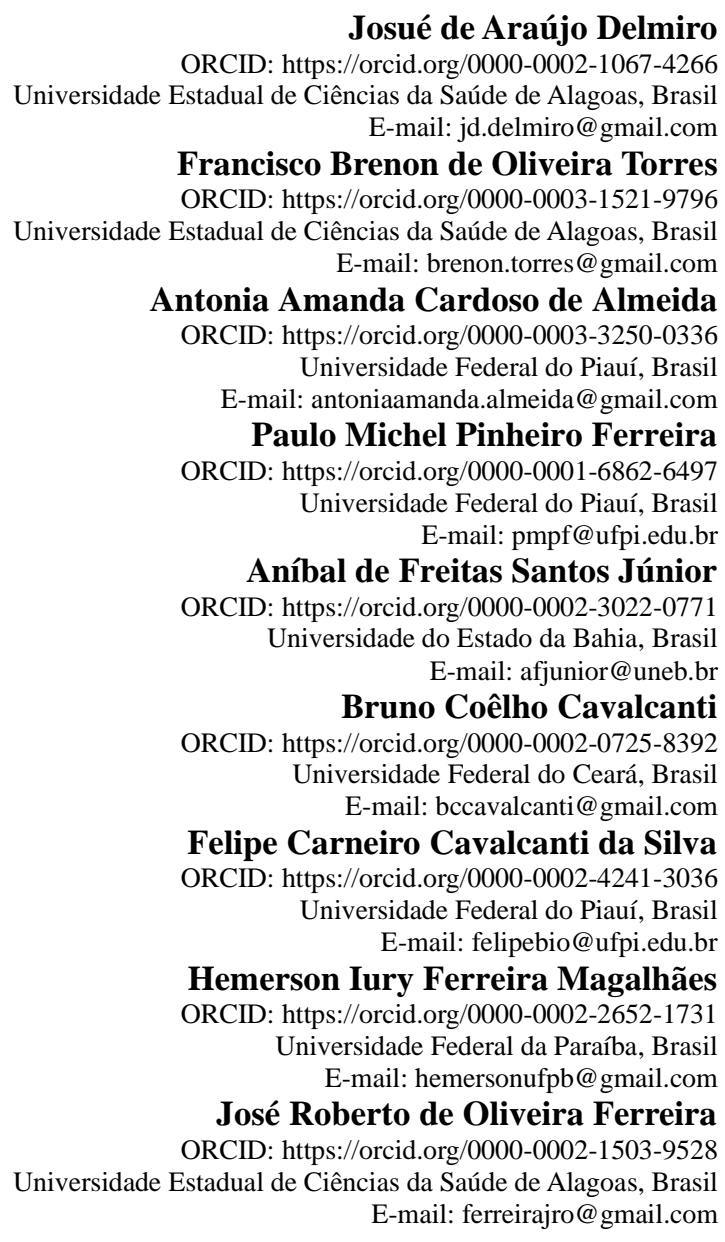

\section{Resumo}

Introdução. Os produtos comestíveis à base de maconha tornaram-se um mercado lucrativo e de grande procura. Apesar de no Brasil não ser legalizada, a maconha é encontrada em preparações orais comercializadas ilegalmente no país e a busca por esses produtos tem crescido nos últimos anos. O presente estudo tem por finalidade reunir dados da literatura científica sobre a fisiologia do sistema endocanabinóide, efeitos do consumo de Cânabis por via oral, farmacocinética, possíveis efeitos tóxicos, terapêuticos e formas de detecção, buscando contribuir para o preenchimento da lacuna ainda existente sobre o tema. Metodologia. Foi realizada revisão integrativa na base de dados PubMed para publicações dos últimos 5 anos em português, inglês e espanhol. Resultados. A pesquisa resultou em 128 artigos os quais foram removidos os trabalhos que não preenchessem os critérios de inclusão, restando 28 artigos utilizados. Conclusões. É necessário que seja realizado um número maior de ensaios clínicos com canabis por via oral, nas diversas formulações existentes, para melhor compreender sua farmacocinética, farmacodinâmica, potencialidades terapêuticas e seus efeitos adversos.

Palavras-chave: Maconha; Canabinoides; Receptor de Canabinoide. 


\begin{abstract}
Introduction. Cannabis-based edibles have become a lucrative and in-demand market. Despite not being legalized in Brazil, marijuana is found in oral preparations illegally marketed in the country and the search for these products has grown in recent years. The present study aims to gather data from the scientific literature on the physiology of the endocannabinoid system, effects of oral cannabis consumption, pharmacokinetics, possible toxic and therapeutic effects and ways of detection, seeking to contribute to filling the gap that still exists on the theme. Methodology. An integrative review was carried out in the PubMed database for publications of the last 5 years in Portuguese, English and Spanish. Results. The search resulted in 128 articles from which the works that did not meet the inclusion criteria were removed, leaving 28 articles used. Conclusions. It is necessary to carry out a greater number of clinical trials with oral cannabis, in the various existing formulations, to better understand its pharmacokinetics, pharmacodynamics, therapeutic potential and its adverse effects.
\end{abstract}

Keywords: Marihuana; Cannabinoids; Cannabinoid Receptor.

\title{
Resumen
}

Introducción. Los comestibles a base de cannabis se han convertido en un mercado lucrativo y en demanda. A pesar de no estar legalizada en Brasil, la marihuana se encuentra en preparaciones orales comercializadas ilegalmente en el país y la búsqueda de estos productos ha crecido en los últimos años. El presente estudio tiene como objetivo recopilar datos de la literatura científica sobre la fisiología del sistema endocannabinoide, efectos del consumo oral de cannabis, farmacocinética, posibles efectos tóxicos y terapéuticos y formas de detección, buscando contribuir a llenar el vacío que aún existe sobre el tema. Metodología. Se realizó una revisión integradora en la base de datos PubMed para publicaciones de los últimos 5 años en portugués, inglés y español. Resultados. La búsqueda resultó en 128 artículos de los cuales se eliminaron los trabajos que no cumplieron con los criterios de inclusión, quedando 28 artículos utilizados. Conclusiones. Es necesario realizar un mayor número de ensayos clínicos con cannabis oral, en las distintas formulaciones existentes, para conocer mejor su farmacocinética, farmacodinamia, potencial terapéutico y sus efectos adversos.

Palabras clave: Marihuana; Cannabinoides; Receptor de Cannabinoides.

\section{Introdução}

Produtos alimentícios com extratos de Cânabis surgiram como um mercado lucrativo em países onde o uso de maconha foi legalizado (White et al., 2020). A procura por esses produtos têm aumentado, uma vez que a curiosidade e o consumo de forma discreta, estimulam a procura (Barros et al, 2016; Boisvert et al., 2020). Além disso, há uma crença que estes sejam mais seguros, isto porque a Cânabis fumada é comparada ao cigarro e fumo comum, que apresentam toxicidade ao pulmão. Todavia, comestíveis com extratos de Cânabis podem ser tão perigosos quanto outras formas de consumo (Leventhal et al., 2020). Nesse sentido, o maior perigo é a lacuna de conhecimento que existe sobre os efeitos sistêmicos do consumo por via oral e formas de detecção (Farmer et al., 2019).

Em um recente estudo prospectivo de patentes de produtos alimentícios com extratos de Cannabis ou com Canabidiol, revelou um crescente aumento do registro de patentes, com a maioria ativa, sendo os maiores detentores países como: China, Estados Unidos e Canadá (Santos et al., 2021). Isto revela a importância do mercado, e que este precisa ser regulado.

Apesar de no Brasil não ser legalizada, é comum encontrar relatos da presença de maconha e/ou seu extrato em alimentos. Nesse sentido, surgi o "Brisadeiro" que é uma versão ilegal do famoso doce brasileiro o "Brigadeiro". No Brisadeiro partes da Cânabis são previamente processadas e tostadas em forno, posteriormente sofrem uma extração a quente com manteiga. Essa manteiga "batizada" é utilizada no preparo do Brisadeiro e outros produtos alimentícios. É importante destacar que o procedimento de aquecimento é necessário para a descarboxilação do Tetra-hidrocanabinol, e, portanto, para a ativação de canabinóides e terpenos através do calor (Barrus et al., 2016).

Em consulta ao Google Trends®, que é uma ferramenta do Google capaz de verificar o quanto determinado termo está sendo pesquisado na internet, conforme Figura 1, a pesquisa pelo "Brisadeiro" tem crescido nos últimos cinco anos. 
Figura 1: Termo de pesquisa "Brisadeiro", filtro aplicado: últimos 05 anos (2016 - 2021), ferramenta Google Trends®.

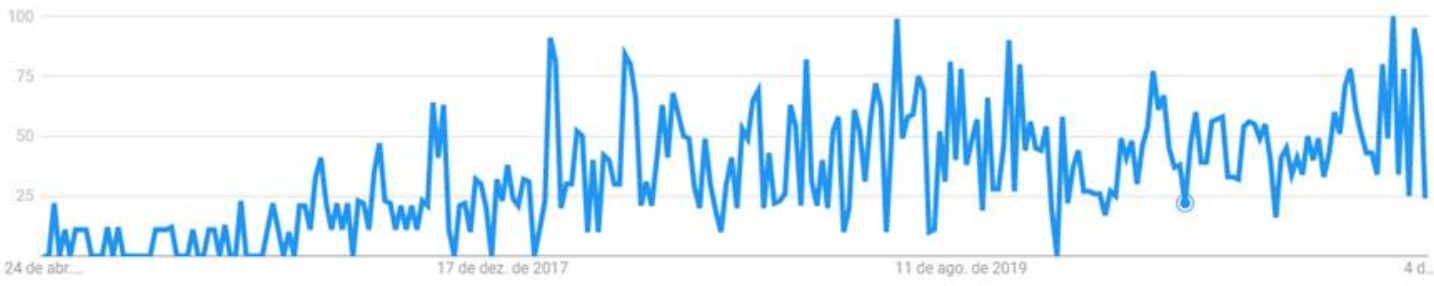

Fonte: Google Trends®.

Nesse contexto, o presente estudo tem por finalidade reunir dados da literatura científica sobre a fisiologia do sistema endocanabinóide, os efeitos do consumo de Cânabis por via oral, sua farmacodinâmica e farmacocinética. Bem como, dados clínicos importantes para a compreensão dos possíveis efeitos tóxicos, terapêuticos e formas de detecção, buscando contribuir para o preenchimento da lacuna de conhecimento existente.

\section{Metodologia}

Foi realizada uma revisão integrativa a partir da base de dados PubMed para publicações dos últimos 5 anos em português, inglês e espanhol. A coleta de dados foi realizada de março a setembro de 2021, com a combinação dos descritores ((Cannabis OR Cannabinoids) AND "Edibles"). Para garantir maior segurança a pesquisa, os descritores selecionados foram controlados por MESH. Definido os descritores, então foram determinados os critérios de inclusão e exclusão. Como critério de inclusão: I - Estudos empíricos, II - Língua inglesa, portuguesa ou espanhola, III- Artigos que buscam avaliar os efeitos de produtos alimentícios à base de extrato de Cannabis ou que analisam os efeitos do consumo por via oral. Enquanto os critérios de exclusão foram: I- Estudos experimentais com animais, II - Estudos in vitro, III - Estudos que abordem outra via de consumo que não seja a oral.

A pesquisa inicial resultou em 128 artigos, estes foram lidos os títulos e resumos disponíveis, sendo retirados 15 artigos por serem revisões e 47 por tratar de outra via que não a oral. Resultando em 66 artigos para leitura completa, destes 1 correspondia a estudos com animais, e 37 a outros assuntos como legislação e estudos de consumo. Restando 28 artigos que foram incluídos no presente trabalho para compor a síntese qualitativa desta revisão integrativa. A estratégia de busca pode ser visualizada na Figura 2. 
Figura 2: Fluxograma da estratégia de busca dos artigos para a revisão integrativa.

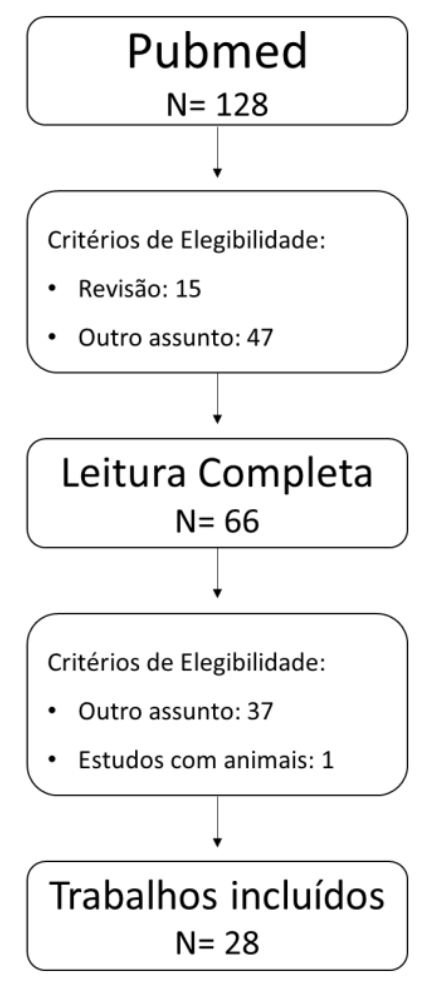

Fonte: Dados da pesquisa (2022).

\section{Aspectos Farmacológicos}

A Cânabis, ou Cannabis, popularmente conhecida como maconha, é uma espécie de planta do gênero Cannabis, que produz mais de 568 compostos únicos, dos quais mais de 100 pertencem à classe dos fitocanabinóides (Figura 2). Estes são moléculas orgânicas com estrutura polifenólica, que exibem uma ampla gama de ações farmacodinâmicas nos receptores canabinoides humanos (Elsohly et al., 2017; Yang et al., 2020).

Figura 3: Estruturas químicas dos principais fitocanabinóides presentes na C.sativa.

\section{(CBD) Canabidiol $\left(\mathrm{C}_{21} \mathrm{H}_{30} \mathrm{O}_{2}\right)$}<smiles>C=C(C)[C@H]1CCC(C)=C[C@H]1c1c(O)cc(CCCCC)cc1O</smiles>

(THC) Tetrahidrocanabinol $\left(\mathrm{C}_{21} \mathrm{H}_{30} \mathrm{O}_{2}\right)$<smiles>CCCCCc1cc(O)c2c(c1)OC(C)(C)[C@H]1CCC(C)=C[C@H]21</smiles>

Fonte: Elaborada pelos autores (Software ACD/ChemSketch) (2022).

\subsection{Mecanismos moleculares de sinalização via receptor canabinoide tipo 1 e tipo 2}

O primeiro receptor canabinoide humano foi identificado em 1990 (Matsuda et al., 1990), sendo então parte de um sistema chamado hoje de sistema endocanabinóide (SECB), descrito como neuromodulador e importante para a manutenção da homeostase (Klumpers \& Thacker, 2019). 
O SECB é compreendido por vários elementos moleculares, como ligantes endógenos denominados de endocanabinóides, receptores, enzimas responsáveis pela hidrólise dos ligantes, e transportadores passivos (Rodríguez et al., 2017), conforme ilustrado na Figura 3.

Figura 4: Estruturas químicas dos endocanabinóides.

Anandamida $\left(\mathrm{C}_{22} \mathrm{H}_{37} \mathrm{NO}_{2}\right)$

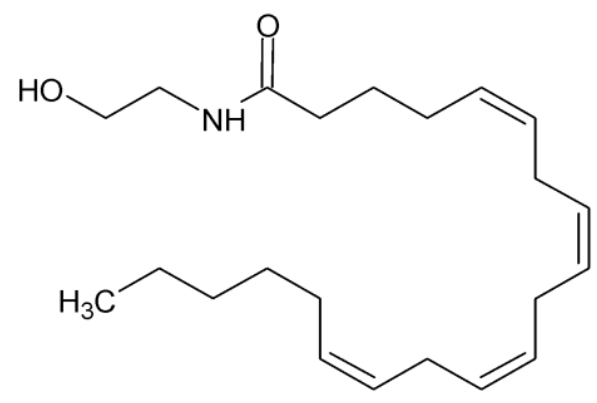

2-Araquinodilglicerol $\left(\mathrm{C}_{23} \mathrm{H}_{38} \mathrm{O}_{4}\right)$

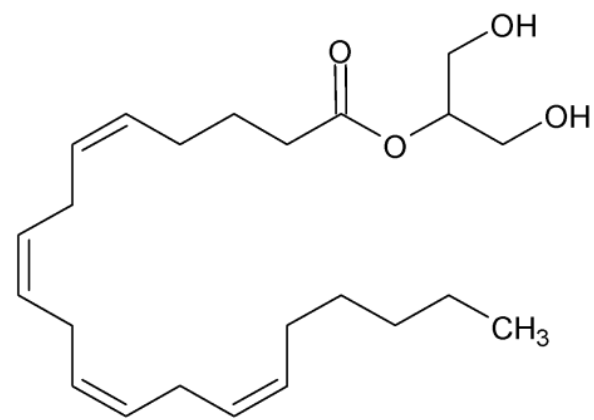

Fonte: Elaborada pelos autores (Software ACD/ChemSketch) (2022).

Até o momento são reconhecidos os receptores canabinóides tipo 01 (CB1) e 2 (CB2), que são do tipo metabotrópico ligados à proteína G (Rodríguez et al., 2017). O CB1 é proeminentemente expresso nos terminais neuronais pré-sinápticos, limitados principalmente ao sistema nervoso central (SNC), e atua na modulação do processamento executivo, emocional, de recompensa e de memória, e sobre os sistemas gabaérgico, dopaminérgico e glutamatérgico. Já os receptores CB2 estão em grande parte confinados ao sistema nervoso periférico e ao sistema imunológico, onde podem influenciar na dor, inflamação e dano tecidual (Wu, 2019; Amin, 2019; Klumpers \& Thacker, 2019).

Dentre os endocanabinoides mais conhecidos estão a anandamida (ANA) e 2-araquinodilglicerol (2-AG). Estes sofrem hidrólise através de enzimas conhecidas como amida hidrolase de ácido graxo (FAAH) e monoacilglicerol lipase (MAGL). Um elemento molecular adicional pertencente ao sistema endocanabinóide é o transportador de membrana de anandamida (AMT), que facilita o movimento da anandamida através da membrana celular (Rodríguez et al., 2017).

Segundo Zou e Kumar (2018), os endocanabinóides ANA e 2-AG são produzidos no terminal pós-sináptico sob demanda em resposta ao aumento da concentração intracelular de cálcio. A ANA é catalisada a partir de $\mathrm{N}$-acilfosfatidiletanolamina (NAPE) através da fosfolipase D específica de NAPE (NAPE-PLD). Já o 2-AG é produzido a partir do diacilglicerol (DAG) pela DAG lipase (DAGL) $\alpha$ ou $\beta$, sendo considerado o ligante primário por estar em maior quantidade no cérebro comparado a ANA (Zou \& Kumar, 2018).

O SECB é um exemplo de sistema retrógrado. Os endocanabinóides são produzidos no terminal pós-sináptico, atravessam prontamente a membrana e viajam de forma retrógrada para ativar os CB1s localizados nos terminais présinápticos. Os CB1s ativados inibem a liberação de neurotransmissores (NT) como GABA e glutamato, por meio da supressão do influxo de cálcio. Já a ANA tem importante função regulatória ao inibir os canais de $\mathrm{Ca}^{2+}$ (cálcio) do tipo L, inibir a produção de 2-AG, e ligar-se nos mesmos receptores CBs como o 2-AG (Zou \& Kumar, 2018) (Figura 4).

Figura 5: Processo de sinalização canabinoide. 


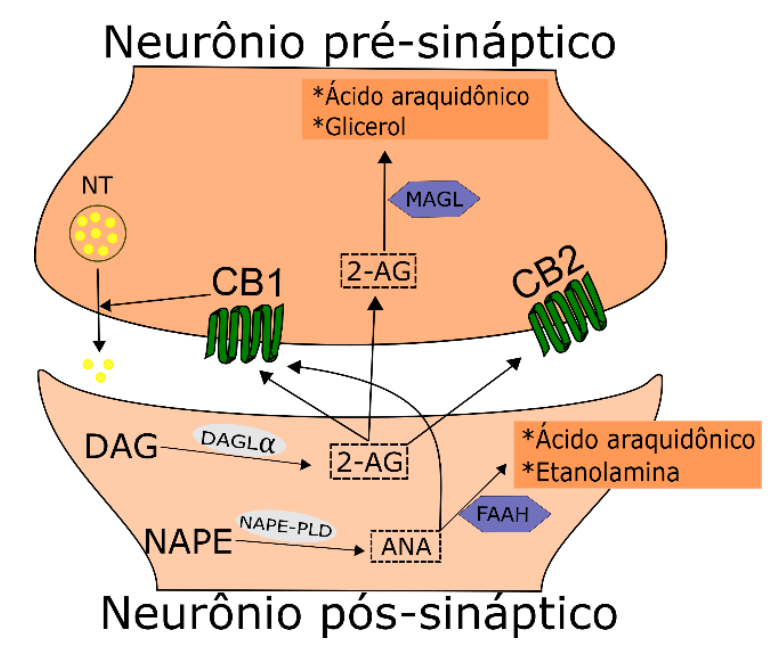

Legenda: Os endocanabinóides 2-AG e ANA são produzidos respectivamente pela DAG e NAPE no neurônio pós-sináptico. São produzidos sob demanda, em seguida são liberados na fenda e de forma anterógrada se ligam nos receptores CB1 e CB2 no neurônio pré-sináptico ligados à proteína G. Esta ligação controla o influxo de cálcio permitindo o controle da liberação de neurotransmissores (NT) na fenda sináptica, como o glutamato e dopamina. A enzima MAGL hidrolisa a 2-AG e a FAAH hidrolisa a ANA finalizando o processo de sinalização. Fonte: Adaptado de Zou \& Kumar, (2018); Rodríguez et al., 2017 (Software Inkscape).

O 2-AG extra na fenda sináptica é absorvido pelos terminais pré-sinápticos e hidrolisado pela monoacilglicerol lipase (MAGL) em ácido araquidônico e glicerol, como também pode ser oxigenado pela ciclooxigenase-2 (COX-2) em ésteres glicerílicos de prostaglandina que regulam a inflamação (Sharkey \& Wiley, 2016). Por outro lado, o ANA é degradado pela amida hidrolase de ácido graxo (FAAH) em ácido araquidônico livre e etanolamina (Joshi, 2019). Já a inibição de FAAH ou MAGL promove um acúmulo endógeno de ANA ou 2-AG, respectivamente, aumentando a neuromodulação (Marzo, 2018).

\subsection{Localização e efeitos no trato gastrointestinal}

O Trato gastrointestinal (TGI) é dotado de receptores canabinóides (CB) e de seus ligantes endógenos que são produzidos localmente de acordo com suas necessidades fisiológicas para reagir rapidamente a distúrbios no intestino e manter a homeostase (Hasenoehrl et al., 2016). Os CB1 estão localizados principalmente nos neurônios mioentéricos e submucosos, além de células epiteliais de cripta e células musculares lisas. Já os CB2 estão localizados principalmente em células epiteliais e inflamatórias, como também presentes em neurônios mioentéricos e submucosos (Camilleri, 2018).

Por meio de vias neuronais e não neuronais, o CB1 modula a motilidade do TGI, a secreção de ácidos gástricos, fluidos, neurotransmissores e hormônios, bem como a permeabilidade do epitélio intestinal (Zou \& Kumar, 2018). O mecanismo de ação dos canabinóides na motilidade intestinal se dá principalmente pela ativação dos receptores CB1 expressos nos neurônios entéricos colinérgicos, deprimindo a liberação de acetilcolina, e consequentemente, causando uma diminuição da contratilidade do músculo liso intestinal (Hasenoehrl et al., 2016).

O Rimonabanto é um potente antagonista competitivo dos receptores canabinóides CB1 no intestino, responsável por aumentar o trânsito gastrointestinal. O contrário é alcançado com o Dronabinol (THC sintético), que causa atrasos no trânsito colônico (Goyal et al., 2017; Camilleri, 2018).

A inibição aguda de enzimas de síntese (DAGL $\propto$ e NAPE-PLD) ou degradação (FAAH ou MAGL) de endocanabinóides também modula a motilidade intestinal (Hasenoehrl et al., 2016). Dismotilidades gastrointestinais foram associadas à expressão ou atividade exacerbada de CB1 e CB2, e a diminuição da atividade entérica de FAAH foi associada à constipação (Maselli \& Camilleri, 2021).

Na inflamação intestinal há aumento da expressão dos receptores CB1, CB2, e da Anandamida, enquanto os níveis de FAAH são reduzidos na fase inicial da colite. Assim, o agonista do receptor CB2 foi mais eficaz em melhorar os limiares de dor de maneira dependente da dose. As preparações de cânabis desempenham um papel anti-inflamatório na doença de Crohn e colite ulcerativa e tem sido usada para tratar anorexia, dor abdominal, vômitos, gastroenterite, diarreia e gastroparesia 
diabética, porém limitada devido aos efeitos psicotrópicos. Já o Canabidiol e Canabigerol compartilham os efeitos benéficos típicos dos canabinóides no intestino sem exercerem quaisquer efeitos psicotrópicos (Goyal et al., 2017; Hasenoehrl et al., 2016).

\section{Estudos Clínicos}

A maioria dos estudos teve como objetivo verificar características farmacocinéticas do Tetra-hidrocanabinol (THC) nos diferentes fluidos corporais, comparando a biodisponibilidade determinada por diferentes vias de administração (Newmeyer et al., 2017; Vandrey et al., 2017). O perfil farmacocinético da cânabis é essencial para compreender e interpretar os testes de toxicologia para detecção de uso recente, testes de drogas e ensaios clínicos de tratamentos (Vandrey et al., 2017).

Outro ponto de destaque nesses estudos, é a busca por formas alternativas de detecção, comparando os níveis no sangue e saliva. Para isso, foram demonstradas diversas maneiras de aferir as concentrações, sendo a maioria na saliva e no sangue. Os trabalhos procuraram estabelecer uma maneira mais fidedigna de aferição pois a forma do consumo difere muito em relação à concentração e os efeitos subjetivos da droga. Os resultados destacam um atraso na biodisponibilidade de THC na corrente sanguínea quando ocorre ingestão por via oral (Newmeyer et al., 2017; Vandrey et al., 2017). Esse atraso na biodisponibilidade pode refletir em casos de intoxicação (Loflin et al., 2017).

Em nenhum deles foi apontada a melhor forma de aferição, sejam para fins policiais, trabalhistas, de competição ou análise terapêutica, mas, foram demonstrados que diversos aspectos, como a forma ingerida (oral ou fumada), o sexo, o tempo de uso, e entre outros aspectos interferem na concentração sérica final de THC (Spindle et al., 2020). Os trabalhos analisados apresentaram várias limitações, entre elas, número de participantes e restrições judiciais quanto ao uso da substância, que restringem muitos estudos.

Na Tabela 1 é apresentada informações sobre estudos clínicos utilizando comestíveis à base de Cânabis encontrados na literatura, levando em consideração os critérios de inclusão e exclusão da pesquisa. 
Tabela 1: Estudos clínicos utilizando comestíveis à base de Cânabis.

\begin{tabular}{|c|c|c|c|c|}
\hline Título & Autoria & Principais objetivos & Materiais e métodos & Resultados/Discussão \\
\hline $\begin{array}{lr}\text { Cannabis Edibles: Blood } \\
\text { and Oral Fluid Cannabinoid } \\
\text { Pharmacokinetics } r \text { and } \\
\text { Evaluation of Oral Fluid } \\
\text { Screening Devices for } \\
\text { Predicting } & \Delta 9- \\
\text { Tetrahydrocannabinol in } \\
\text { Blood and Oral Fluid } \\
\text { following Cannabis } \\
\text { Brownie Administration }\end{array}$ & $\begin{array}{l}\text { Newmeyer et } \\
\text { al., } 2017\end{array}$ & $\begin{array}{l}\text { Predizer a concentração de 9-Tetra- } \\
\text { hidrocanabinol no sangue e fluido oral após o } \\
\text { consumo de Brownie de Cânabis, através de } \\
\text { métodos de triagem. } \\
\begin{array}{l}\text { Estabelecer um método padrão de } \\
\text { identificação da concentração de } \\
\text { canabinóides, pela concentração sanguínea ou } \\
\text { saliva }\end{array}\end{array}$ & $\begin{array}{l}\text { Adultos de } 18-50 \text { anos com autorrelato de } \\
\text { uso de Cânabis, onde foi administrado } \\
\text { uma dose inteira de cânabis oral em } \\
\text { preparo de Brownie e ficando em unidade } \\
\text { de pesquisa por } 48 \text { horas, sendo coletadas } \\
\text { amostras de sangue e da saliva em } \\
\text { preparos específicos }\end{array}$ & $\begin{array}{l}\text { Todos os participantes da pesquisa testaram positivo para THC } \\
\text { por até } 5 \text { horas após a dose oral administrada } \\
\text { É possível identificar derivados canabinoides em um nível de } \\
\text { concentração máxima de } 0,8 \text { a } 8 \text { horas após a vaporização, } \\
\text { enquanto que por via oral é possível detectar entre } 1 \text { e } 5 \text { horas } \\
\text { mais tarde. }\end{array}$ \\
\hline $\begin{array}{l}\text { Subjective and } \\
\text { physiological effects, and } \\
\text { expired carbon monoxide } \\
\text { concentrations in frequent } \\
\text { and occasional cannabis } \\
\text { smokers following smoked, } \\
\text { vaporized, and oral } \\
\text { cannabis administration }\end{array}$ & $\begin{array}{l}\text { Newmeyer et } \\
\text { al., } 2017\end{array}$ & $\begin{array}{l}\text { Comparar a quantidade de monóxido de } \\
\text { carbono expirada, no ato de fumar, comer ou } \\
\text { vaporizar produtos de cânabis. } \\
\text { Aferir e comparar medidas fisiológicas }\end{array}$ & $\begin{array}{l}\text { Adultos de } 18 \text { a } 50 \text { anos foram submetidos } \\
\text { ao ensaio com a administração de cânabis } \\
\text { na forma oral, fumada ou vaporizada, ou } \\
\text { administração de placebo, e verificados os } \\
\text { objetivos. }\end{array}$ & $\begin{array}{l}\text { Maior concentração do sangue na cânabis fumada em comparação } \\
\text { com a oral, devido à degradação do THC no ácido gástrico. } \\
\text { O nível sérico de } 11-\mathrm{OH}-\mathrm{THC} \text { é maior quando consumido de } \\
\text { forma oral em comparação a forma isolada }\end{array}$ \\
\hline 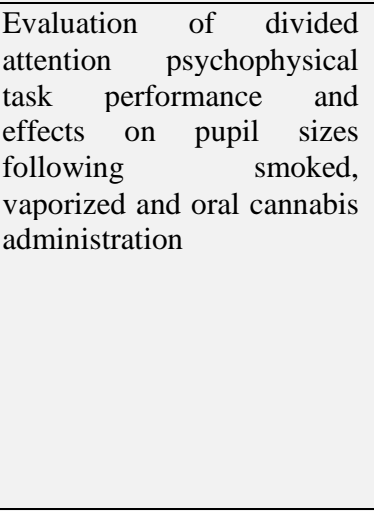 & $\begin{array}{l}\text { Newmeyer et } \\
\text { al., } \\
2017\end{array}$ & $\begin{array}{l}\text { Comparar aspectos psicofísicos da atenção } \\
\text { dividida, do desempenho em tarefas e } \\
\text { tamanho das pupilas, na maconha fumada, } \\
\text { oral e vaporizada. }\end{array}$ & $\begin{array}{l}\text { Os participantes consumiram cânabis oral, } \\
\text { fumada ou vaporizada por } 10 \text { minutos, } \\
\text { sendo coletada uma amostra de sangue } \\
\text { antes e uma outra amostra } 72 \mathrm{~h} \text { após o } \\
\text { consumo. }\end{array}$ & $\begin{array}{l}\text { Em todas as formas de consumo foram identificados problemas de } \\
\text { condução e direção. } \\
\text { No consumo oral foi observado um maior risco de psicose, sem } \\
\text { mecanismo conhecido. } \\
\text { Não foram observadas alterações muito significativas no teste de } \\
\text { Romberg (equilíbrio). } \\
\text { Em todas as formas houve alteração do andar e caminhar, sendo } \\
\text { mais acentuado nos usuários ocasionais e mais relacionado ao } \\
\text { consumo oral. } \\
\text { O tamanho médio das pupilas após } 1,5 \text { e } 3,5 \mathrm{~h} \text { foi de } 3,3 \mathrm{~mm} \text { e } \\
3,4 \text { mm após o placebo, comparado a } 3,6 \mathrm{~mm} \text { e } 3,8 \mathrm{~mm} \text { com o uso } \\
\text { de cânabis oral. }\end{array}$ \\
\hline $\begin{array}{l}\text { Placebo Effects of Edible } \\
\text { Cannabis: } \quad \text { Reported } \\
\text { Intoxication Effects at a } 30- \\
\text { Minute Delay }\end{array}$ & $\begin{array}{l}\text { Loflin et al., } \\
2017\end{array}$ & $\begin{array}{l}\text { Relatar os efeitos do cânabis comestível } \\
\begin{array}{l}\text { Determinar a intoxicação por formas } \\
\text { comestíveis de cânabis }\end{array}\end{array}$ & $\begin{array}{l}\text { Os participantes receberam de forma } \\
\text { aleatória um pirulito placebo, um com } \\
\text { altas doses e outro com baixas doses de } \\
\text { tetrahidrocanabinol (THC) e após isso } \\
\text { foram coletados os auto relatos e aferidas } \\
\text { as alterações. }\end{array}$ & $\begin{array}{l}\text { Foi notado efeito placebo em pessoas que não receberam cânabis } \\
\text { quando informado que havia THC na amostra. } \\
\text { O principal efeito foi a diminuição do humor negativo retornando } \\
\text { a linha de base } 60 \text { minutos após. } \\
\text { Foi notado início de ação dos efeitos } 30 \text { min após a ingestão em } \\
\text { alguns participantes, e, em outros, de } 90 \text { a } 120 \text { min após a } \\
\text { ingestão. }\end{array}$ \\
\hline $\begin{array}{l}\text { Pharmacokinetic Profile of } \\
\text { Oral Cannabis }\end{array}$ & $\begin{array}{l}\text { Vandrey et al., } \\
2017\end{array}$ & $\begin{array}{l}\text { Caracterizar a farmacocinética de Cânabis } \\
\text { após a administração oral. }\end{array}$ & $\begin{array}{l}\text { Participantes entre } 18 \text { e } 45 \text { anos com } \\
\text { histórico de uso de cânabis, mas sem }\end{array}$ & $\begin{array}{l}\text { O estudo conclui que há uma diferença drástica na concentração } \\
\text { de THC na saliva, quando comparamos a via de administração de }\end{array}$ \\
\hline
\end{tabular}


in Humans: Blood and Oral

Fluid Disposition

and Relation to

Pharmacodynamic

Outcomes

\section{ton}

Pharmacokinetics

\begin{tabular}{lrl}
\hline Pharmacokinetics & of & Spindle et al., \\
Cannabis Brownies: & A & 2020
\end{tabular}

Cannabis Brownies: A
Controlled Examination of

$\Delta 9$-Tetrahydrocannabinol

and Metabolites in Blood

and Oral Fluid of Healthy

Adult Males

and Females utilizar ela e outras substâncias ilícitas nos

3 meses anteriores ao estudo. Um total

18 participantes, entre eles 9 homens e 9

mulheres. Foram ofertadas 3 doses

diferentes de THC, 10, 24 e $50 \mathrm{mg}$. Foram

coletadas amostras de sangue, urina, saliva

ao longo de 9 dias, e uma amostra de

cabelo no $1^{\circ}$ e $9^{\circ}$ dia, sendo realizados os

testes farmacocinéticos.

Relatar a farmacocinética de THC em adultos através de um estudo controlado usando cânabis comestível.

Verificar a variabilidade interindividual na absorção e eliminação de THC.
Os participantes ingeriram 4 doses separadas de Cânabis $(0,10,25$ e $50 \mathrm{mg}$ THC) com uma semana de intervalo entre elas. Além disso, os participantes se

abstiveram do uso de Cânabis por 2 mese antes do estudo. Foram coletadas amostras de sangue e saliva para estudo da farmacocinética do THC.
Cânabis, se comestível ou fumada, sendo a primeira forma de apresentação a que apresentou as maiores concentrações da substância na saliva.

Os participantes relatam efeitos subjetivos da droga apenas com a ingestão de mais de $25 \mathrm{mg}$, mesmo o estudo mostrando concentrações séricas suficientes para provar efeito nas 3 doses administradas.

O tempo médio de duração dos efeitos da droga foram maiores na forma oral em comparação à fumada.

A concentração máxima de THC sanguíneo demorou mais para ser alcançada quando cânabis era consumida de forma oral quando comparado ao fumado.

A concentração de THC na saliva foi muito maior que a concentração sanguínea.

Mulheres apresentaram maiores concentrações de canabinóides no sangue em comparação aos homens, quando administrada a mesma dose na forma oral.

Fonte: Autores (2022) 


\section{Efeitos Gerais e Intoxicação}

Os efeitos gerais da cânabis comestível podem ser divididos em efeitos subjetivos positivos, como sentimentos agradáveis, felicidade, relaxamento, energia, aumento do desejo sexual, criatividade, social, e efeitos negativos, como dificuldade de concentração, ansiedade, sonolência, vertigem, perda de controle, preguiça e sintomas gastrointestinais. Além disso, há o fator de gravidade que pode trazer riscos à saúde, como condução prejudicada e transtornos psiquiátricos (Boisvert et al., 2020).

O tetrahidrocanabinol em altas doses pode causar psicose dose-dependente aguda e transitória com sintomas positivos e negativos da esquizofrenia, como também pode aumentar o risco de desenvolver esquizofrenia propriamente dita em até duas vezes (Volkowet al., 2016). Embora os efeitos negativos da cânabis sejam bem conhecidos para algumas condições, como a exacerbação da psicose associada ao THC, outros provavelmente surgirão à medida que mais pacientes forem expostos à cânabis, como interações medicamentosas negativas, ao exemplo do aumento mediado pelo THC no tempo de sangramento em pacientes em uso de varfarina (Volkow \& Ruben, 2019).

As preparações comestíveis de cânabis podem resultar em riscos maiores que as preparações inaláveis devido à lenta taxa de absorção do THC ingerido por via oral. O pico do nível sanguíneo do THC inalado e os efeitos da droga são alcançados em cerca de 30 minutos, diferindo dos comestíveis, que são alcançados em aproximadamente 3 horas. Isto torna mais difícil para os usuários de cânabis comestível titular as doses necessárias para atingir os efeitos desejados da droga, além do que a depuração mais lenta do THC oral pode levar ao acúmulo da droga se os usuários tomarem doses adicionais quando não sentirem os efeitos desejados tão rapidamente quanto esperado (Volkow \& Ruben, 2019).

\section{Perspectivas Terapêutica de Comestíveis à Base de Canabis}

Para averiguar os novos estudos que buscam avaliar clinicamente os efeitos do consumo de comestíveis a base de Cânabis foi realizado um levantamento no site Clinical Trials do governo americano. Foram encontrados quatro ensaios clínicos que estão ainda no processo de recrutamento de voluntários para o estudo. Interessantemente, estes estudos buscam avaliar os possíveis efeitos terapêuticos de comestíveis à base de Cânabis em diversos processos patológicos, como câncer, ansiedade, inflamação e dor crônica.

O primeiro estudo identificado como "NCT03491384", busca compreender os efeitos ansiolíticos, cognitivos, e propriedades anti-inflamatórias da cânabis em pacientes com ansiedade leve a moderada, após ingestão de alimentos à base de maconha e flor defumada. O segundo estudo "NCT03522103", busca examinar a influência da cânabis no estado mental e físico dos pacientes com base na proporção de THC ou CBD, diferenciando o consumo por via oral ou inalatória. O estudo “NCT03617692” é observacional entre pacientes com câncer que já consomem alimentos à base de Cânabis como forma de tratamento dos sintomas da doença, e verifica os efeitos agudos do produto no quadro. Por fim, o estudo "NCT03522324" observa os efeitos de comestíveis a base de Cânabis no alívio da dor, inflamação e disfunção cognitiva, nos diferentes níveis de THC e CBD no sangue em pacientes com dor crônica.

Nenhum destes estudos possui resultados divulgados ainda, porém espera-se que a contribuição deles traga esclarecimentos sobre o uso de comestíveis a base de Cânabis como recursos terapêuticos no tratamento das mais diversas patologias.

\section{Considerações Finais}

De acordo com a pesquisa realizada, é necessário que sejam realizados um número maior de ensaios clínicos com canabis por via oral, nas diversas formulações existentes, para melhor compreender sua farmacocinética e potencialidades. 
Atualmente, alguns estudos clínicos buscam investigar o potencial terapêutico de produtos alimentícios com extratos de canabis. Outro ponto, é a urgência de padronização nos comestíveis de canabis, somente assim será possível explorar todo seu potencial terapêutico.

Os ensaios clínicos com produtos alimentícios à base de extratos de Cannabis são promissores, porém carecem ainda de padronização. Cada produto é feito, na maioria das vezes, de forma artesanal, com extratos de Cannabis preparados de forma e com condições diferentes. Mesmo a forma de quantificar metabólitos difere. É preciso trabalhar com extratos padronizados que permitam a comparação de diversos estudos. Nesse sentido, a criação de extratos padronizados possibilitará avanços significativos na pesquisa com produtos alimentícios à base de Cannabis, possibilitando a expansão do mercado, e desenvolvimento de produtos alimentícios terapêuticos à base de Cannabis.

\section{Agradecimentos}

Os autores agradecem o apoio recebido pelo programa de iniciação científica da Universidade Estadual de Ciências da Saúde de Alagoas (Uncisal), Conselho Nacional de Desenvolvimento Científico e Tecnológico (CNPq) e a Fundação de Amparo à Pesquisa do Estado de Alagoas (Fapeal).

\section{Referências}

Amin, M. R., \& Ali, D. W. (2019). Recent Advances in Cannabinoid Physiology and Pathology. Pharmacology of medical cannabis, $151-165$.

Barrus, D. G., Capogrossi, K. L., Cates, S. C., Gourdet, C. K., Peiper, N. C., Novak, S. P., Lefever, T. W., \& Wiley, J. L. (2016). Tasty THC: Promises and Challenges of Cannabis Edibles. Methods report (RTI Press), 2016.

Ewusi Boisvert, E., Bae, D., Pang, R. D., Davis, J. P., Kelley-Quon, L. I., Barrington-Trimis, J. L., Kirkpatrick, M. G., Chai, S. H., \& Leventhal, A. M. (2020). Subjective effects of combustible, vaporized, and edible cannabis: Results from a survey of adolescent cannabis users. Drug and alcohol dependence, 206, 107716.

Camilleri, M. (2018). Cannabinoids and gastrointestinal motility: Pharmacology, clinical effects, and potential therapeutics in humans. Neurogastroenterology and motility: the official journal of the European Gastrointestinal Motility Society, 30(9), e13370.

Cohen, K., Weizman, A., \& Weinstein, A. (2019). Positive and Negative Effects of Cannabis and Cannabinoids on Health. Clinical pharmacology and therapeutics, 105(5), 1139-1147.

Di Marzo V. (2018). New approaches and challenges to targeting the endocannabinoid system. Nature reviews. Drug discovery, 17(9), 623-639.

ElSohly, M. A., Radwan, M. M., Gul, W., Chandra, S., \& Galal, A. (2017). Phytochemistry of Cannabis sativa L. Progress in the chemistry of organic natural products, 103, 1-36.

Farmer, S., Slavin, M. N., Loflin, M., Luba, R., \& Earleywine, M. (2019). Aversiveness and Meaningfulness of Uncomfortable Experiences with Edible Cannabis. Journal of psychoactive drugs, 51(5), 413-420.

Goyal, H., Singla, U., Gupta, U., \& May, E. (2017). Role of cannabis in digestive disorders. European journal of gastroenterology \& hepatology, 29(2), 135143.

Hasenoehrl, C., Taschler, U., Storr, M., \& Schicho, R. (2016). The gastrointestinal tract - a central organ of cannabinoid signaling in health and disease. Neurogastroenterology and motility : the official journal of the European Gastrointestinal Motility Society, 28(12), 1765-1780.

Joshi, N., \& Onaivi, E. S. (2019). Endocannabinoid System Components: Overview and Tissue Distribution. Advances in experimental medicine and biology, $1162,1-12$.

Klumpers, L. E., \& Thacker, D. L. (2019). A Brief Background on Cannabis: From Plant to Medical Indications. Journal of AOAC International, 102(2), 412420 .

Leventhal, A. M., Bae, D., Kechter, A., \& Barrington-Trimis, J. L. (2020). Psychiatric comorbidity in adolescent use and poly-use of combustible, vaporized, and edible cannabis products. Journal of psychiatric research, 124, 91-98.

Loflin, M., Earleywine, M., Farmer, S., Slavin, M., Luba, R., \& Bonn-Miller, M. (2017). Placebo Effects of Edible Cannabis: Reported Intoxication Effects at a 30-Minute Delay. Journal of psychoactive drugs, 49(5), 393-397.

Maselli, D. B., \& Camilleri, M. (2021). Pharmacology, Clinical Effects, and Therapeutic Potential of Cannabinoids for Gastrointestinal and Liver Diseases. Clinical gastroenterology and hepatology : the official clinical practice journal of the American Gastroenterological Association, $19(9), 1748-1758 . e 2$. 
Research, Society and Development, v. 11, n. 2, e10711225422, 2022

(CC BY 4.0) | ISSN 2525-3409 | DOI: http://dx.doi.org/10.33448/rsd-v11i2.25422

Matsuda, L. A., Lolait, S. J., Brownstein, M. J., Young, A. C., \& Bonner, T. I. (1990). Structure of a cannabinoid receptor and functional expression of the cloned cDNA. Nature, 346(6284), 561-564.

Murillo-Rodriguez, E., Pastrana-Trejo, J. C., Salas-Crisóstomo, M., \& de-la-Cruz, M. (2017). The Endocannabinoid System Modulating Levels of Consciousness, Emotions and Likely Dream Contents. CNS \& neurological disorders drug targets, 16(4), 370-379.

Newmeyer, M. N., Swortwood, M. J., Andersson, M., Abulseoud, O. A., Scheidweiler, K. B., \& Huestis, M. A. (2017). Cannabis Edibles: Blood and Oral Fluid Cannabinoid Pharmacokinetics and Evaluation of Oral Fluid Screening Devices for Predicting $\Delta 9$-Tetrahydrocannabinol in Blood and Oral Fluid following Cannabis Brownie Administration. Clinical chemistry, 63(3), 647-662.

Newmeyer, M. N., Swortwood, M. J., Taylor, M. E., Abulseoud, O. A., Woodward, T. H., \& Huestis, M. A. (2017). Evaluation of divided attention psychophysical task performance and effects on pupil sizes following smoked, vaporized and oral cannabis administration. Journal of applied toxicology : $J A T, 37(8), 922-932$.

Newmeyer, M. N., Swortwood, M. J., Abulseoud, O. A., \& Huestis, M. A. (2017). Subjective and physiological effects, and expired carbon monoxide concentrations in frequent and occasional cannabis smokers following smoked, vaporized, and oral cannabis administration. Drug and alcohol dependence, $175,67-76$

Santos, D. E. L., Santos, M. S. V., Florêncio, M. N. da S., \& Oliveira, J. A. M. de. (2021). Technological patenting prospecting of food products with Cannabis sativa or cannabidiol (CBD) addition. Research, Society and Development, 10(4), e20210413929.

Spindle, T. R., Cone, E. J., Herrmann, E. S., Mitchell, J. M., Flegel, R., LoDico, C., Bigelow, G. E., \& Vandrey, R. (2020). Pharmacokinetics of Cannabis Brownies: A Controlled Examination of $\Delta 9$-Tetrahydrocannabinol and Metabolites in Blood and Oral Fluid of Healthy Adult Males and Females. Journal of analytical toxicology, 44(7), 661-671.

Vandrey, R., Herrmann, E. S., Mitchell, J. M., Bigelow, G. E., Flegel, R., LoDico, C., \& Cone, E. J. (2017). Pharmacokinetic Profile of Oral Cannabis in Humans: Blood and Oral Fluid Disposition and Relation to Pharmacodynamic Outcomes. Journal of analytical toxicology, 41(2), 83-99.

Volkow, N. D., Swanson, J. M., Evins, A. E., DeLisi, L. E., Meier, M. H., Gonzalez, R., Bloomfield, M. A., Curran, H. V., \& Baler, R. (2016). Effects of Cannabis Use on Human Behavior, Including Cognition, Motivation, and Psychosis: A Review. JAMA psychiatry, 73(3), 292-297.

Volkow, N. D., \& Baler, R. (2019). Emergency Department Visits From Edible Versus Inhalable Cannabis. Annals of internal medicine, 170(8), 569-570.

White, A. E., Van Tubbergen, C., Raymes, B., Contreras, A. E., \& Scallan Walter, E. J. (2020). Cannabis-Infused Edible Products in Colorado: Food Safety and Public Health Implications. American journal of public health, 110(6), 790-795.

Wu J. (2019). Cannabis, cannabinoid receptors, and endocannabinoid system: yesterday, today, and tomorrow. Acta pharmacologica Sinica, 40(3), 297-299.

Yang, Y., Vyawahare, R., Lewis-Bakker, M., Clarke, H. A., Wong, A., \& Kotra, L. P. (2020). Bioactive Chemical Composition of Cannabis Extracts and Cannabinoid Receptors. Molecules (Basel, Switzerland), 25(15), 3466.

Zou, S., \& Kumar, U. (2018). Cannabinoid Receptors and the Endocannabinoid System: Signaling and Function in the Central Nervous System. International journal of molecular sciences, 19(3), 833. 